\title{
Phase Transition of the Higher Dimensional Charged Gauss-Bonnet Black Hole in de Sitter Spacetime
}

\author{
Meng-Sen Ma, ${ }^{1,2}$ Li-Chun Zhang, ${ }^{1,2}$ Hui-Hua Zhao, ${ }^{1,2}$ and Ren Zhao ${ }^{1,2}$ \\ ${ }^{1}$ Department of Physics, Shanxi Datong University, Datong 037009, China \\ ${ }^{2}$ Institute of Theoretical Physics, Shanxi Datong University, Datong 037009, China \\ Correspondence should be addressed to Meng-Sen Ma; mengsenma@gmail.com
}

Received 26 November 2014; Revised 21 February 2015; Accepted 23 February 2015

Academic Editor: Chao-Qiang Geng

Copyright (c) 2015 Meng-Sen Ma et al. This is an open access article distributed under the Creative Commons Attribution License, which permits unrestricted use, distribution, and reproduction in any medium, provided the original work is properly cited. The publication of this article was funded by $\mathrm{SCOAP}^{3}$.

\begin{abstract}
We study the phase transition of charged Gauss-Bonnet-de Sitter (GB-dS) black hole. For black holes in de Sitter spacetime, there is not only black hole horizon, but also cosmological horizon. The thermodynamic quantities on both horizons satisfy the first law of the black hole thermodynamics, respectively; moreover, there are additional connections between them. Using the effective temperature approach, we obtained the effective thermodynamic quantities of charged GB-dS black hole. According to Ehrenfest classification, we calculate some response functions and plot their figures, from which one can see that the spacetime undergoes a second-order phase transition at the critical point. It is shown that the critical values of effective temperature and pressure decrease with the increase of the value of GB parameter $\alpha$.
\end{abstract}

\section{Introduction}

Due to the existence of Hawking radiation and the entropy, black holes may display themselves like thermodynamic systems [1-4]. Phase transitions and critical phenomena are important characteristics of ordinary thermodynamic system. Thus, the natural question to ask is whether there also exists phase transition in the black hole thermodynamics. The pioneer work of Hawking and Page gives us an definitive answer [5]. Recently, the phase transitions of black holes in asymptotically anti-de Sitter (AdS) spacetime have received considerable attention [6-17]. In particular, by considering the cosmological constant as thermodynamic pressure $P=$ $-\Lambda / 8 \pi=(d-1)(d-2) / 16 \pi l^{2}$, one can introduce an extended phase space, in which the $P-V$ criticality has been extensively discussed [18-35]. It is shown that many black holes in AdS spacetime exhibit similar critical behaviors to those of van der Waals liquid-gas system.

The astronomical observations show that our universe is probably an asymptotically de Sitter (dS) one. It raises the interest on black holes in dS spacetime. There are many works on the thermal properties of black holes in de Sitter (dS) spacetime [36-48]. As is well known, there are multihorizons for black holes in dS spacetime. On the black hole event horizon and the cosmological horizon, there are different temperatures, which prevent the black hole thermodynamic system in equilibrium. It should be noted that there are common parameters $M, Q$, and $\Lambda$ in the thermodynamic quantities corresponding to the black hole horizon to the cosmological horizon. Thus, these thermodynamic quantities are not independent. Taking into account their connections is relevant to the understanding of thermal properties of $\mathrm{dS}$ spacetime.

Higher derivative curvature terms occur in many occasions, such as in the semiclassically quantum gravity and in the effective low-energy action of superstring theories. Among the many theories of gravity with higher derivative curvature terms, due to the special features, the GaussBonnet (GB) gravity has attracted much interest. The thermodynamic properties and phase structures of GB-AdS black 
hole have been briefly discussed in [49]. In [26-29], the critical phenomena and phase transition of the charged GBAdS black hole have been studied extensively.

In this paper, we study the thermal properties of charged GB-dS black hole after considering the connections between the black hole horizon and the cosmological horizon. Using the effective equilibrium temperature approach, we calculate some effective thermodynamic quantities, from which we can analyze whether there exists phase transition for charged GBdS black hole.

The paper is arranged as follows: in Section 2, we simply introduce the charged GB-dS black hole. In Section 3, we will calculate the effective thermodynamic quantities. In Section 4 , we derive the heat capacity at constant pressure, the volume expansivity $\beta$, and the isothermal compressibility $\kappa$ and plot some curves to demonstrate the phase transition. We will make some concluding remarks in Section 5 (we use the units $G_{d}=\hbar=c=k_{B}$ $=1)$.

\section{Charged GB-dS Black Hole}

The action of the $d$-dimensional Einstein-Gauss-BonnetMaxwell-dS theory has the form

$$
\begin{aligned}
S=\frac{1}{16 \pi} \int d^{d} x \sqrt{-g}[ & R-2 \Lambda \\
+ & \alpha\left(R_{\mu \nu \gamma \delta} R^{\mu \nu \gamma \delta}-4 R_{\mu \nu} R^{\mu \nu}+R^{2}\right) \\
& \left.-4 \pi F_{\mu \nu} F^{\mu \nu}\right],
\end{aligned}
$$

where $\alpha$ is the GB coupling constant, the cosmological constant is $\Lambda=(d-1)(d-2) / 2 l^{2}$ for dS spacetime, and $F_{\mu \nu}$ is the Maxwell field strength. The $d$-dimensional static charged GB-dS black hole solution for the action is described by

$$
\begin{aligned}
d s^{2}= & -f d t^{2}+f^{-1} d r^{2} \\
& +r^{2}\left(d \theta^{2}+\sin ^{2} d \phi^{2}+\cos ^{2} \theta d \Omega_{d-4}^{2}\right),
\end{aligned}
$$

with the metric function given by $[26,27,33]$

$$
f(r)=1+\frac{r^{2}}{2 \widetilde{\alpha}}\left(1-\sqrt{1+\frac{64 \pi \widetilde{\alpha} M}{(d-2) \Sigma_{k} r^{d-1}}-\frac{2 \widetilde{\alpha} Q^{2}}{(d-2)(d-3) r^{2 d-4}}+\frac{8 \widetilde{\alpha} \Lambda}{(d-1)(d-2)}}\right),
$$

where $\widetilde{\alpha}=(d-3)(d-4) \alpha, \Sigma_{k}$ is the area of a unite $(d-2)$ dimensional sphere, $M$ is the black hole mass, and $Q$ is related to the charge of the black hole. Horizons occur where $f(r)=$ 0 , the largest root is the cosmological horizon $r=r_{c}$, and the next root is the black hole event horizon $r=r_{+}$. When $M=Q=0$, solution (2) reduces to the pure GB-dS space with only one cosmological horizon at $r_{c}=l_{c}$.

The equations $f\left(r_{+}\right)=0$ and $f\left(r_{c}\right)=0$ are rearranged to br as follows:

$$
\begin{aligned}
M= & \frac{(d-2) \Sigma_{k} r_{+}^{d-3}}{16 \pi}\left(1+\frac{\tilde{\alpha}}{r_{+}^{2}}\right)-\frac{\Sigma_{k} r_{+}^{d-1} \Lambda}{8 \pi(d-1)} \\
& +\frac{\Sigma_{k} Q^{2}}{32 \pi(d-3) r_{+}^{d-3}}, \\
M= & \frac{(d-2) \Sigma_{k} r_{c}^{d-3}}{16 \pi}\left(1+\frac{\tilde{\alpha}}{r_{c}^{2}}\right)-\frac{\Sigma_{k} r_{c}^{d-1} \Lambda}{8 \pi(d-1)} \\
& +\frac{\Sigma_{k} Q^{2}}{32 \pi(d-3) r_{c}^{d-3}},
\end{aligned}
$$

from which one can derive

$$
\begin{aligned}
M= & \frac{(d-2) \Sigma_{k} r_{+}^{d-3} r_{c}^{d-3}\left(r_{c}^{2}-r_{+}^{2}\right)}{16 \pi\left(r_{c}^{d-1}-r_{+}^{d-1}\right)}\left(1+\frac{\widetilde{\alpha}\left(r_{c}^{2}+r_{+}^{2}\right)}{r_{c}^{2} r_{+}^{2}}\right) \\
& +\frac{\Sigma_{k} Q^{2}\left(r_{c}^{2 d-4}-r_{+}^{2 d-4}\right)}{32 \pi(d-3) r_{c}^{d-3} r_{+}^{d-3}\left(r_{c}^{d-1}-r_{+}^{d-1}\right)}
\end{aligned}
$$

by eliminating the $\Lambda$, and

$$
\begin{aligned}
\Lambda= & \frac{(d-1)(d-2)}{2\left(r_{c}^{d-1}-r_{+}^{d-1}\right)}\left(r_{c}^{d-3}-r_{+}^{d-3}+\widetilde{\alpha}\left(r_{c}^{d-5}-r_{+}^{d-5}\right)\right) \\
& -\frac{(d-1) Q^{2}\left(r_{c}^{d-3}-r_{+}^{d-3}\right)}{4(d-3) r_{c}^{d-3} r_{+}^{d-3}\left(r_{c}^{d-1}-r_{+}^{d-1}\right)}
\end{aligned}
$$

by eliminating $M$.

The surface gravities of black hole horizon and the cosmological horizon are

$$
\begin{aligned}
\kappa_{+}= & \left.\frac{f^{\prime}(r)}{2}\right|_{r=r_{+}} \\
= & -\frac{1}{r_{+}}+\frac{(d-1) r_{c}^{d-3} r_{+}\left(r_{c}^{2}-r_{+}^{2}\right)}{2\left(r_{+}^{2}+2 \widetilde{\alpha}\right)\left(r_{c}^{d-1}-r_{+}^{d-1}\right)}\left(1+\frac{\widetilde{\alpha}\left(r_{c}^{2}+r_{+}^{2}\right)}{r_{c}^{2} r_{+}^{2}}\right) \\
& -\frac{Q^{2}}{4(d-3)\left(r_{+}^{2}+2 \widetilde{\alpha}\right) r_{+}^{2 d-7}} \\
& \cdot\left(2-\frac{(d-1)\left(r_{c}^{2 d-4}-r_{+}^{2 d-4}\right)}{(d-2) r_{c}^{d-3}\left(r_{c}^{d-1}-r_{+}^{d-1}\right)}\right), \\
\kappa_{c}= & \left.\frac{f^{\prime}(r)}{2}\right|_{r=r_{c}} \\
= & -\frac{1}{r_{c}}+\frac{(d-1) r_{+}^{d-3} r_{c}\left(r_{c}^{2}-r_{+}^{2}\right)}{2\left(r_{c}^{2}+2 \widetilde{\alpha}\right)\left(r_{c}^{d-1}-r_{+}^{d-1}\right)}\left(1+\frac{\widetilde{\alpha}\left(r_{c}^{2}+r_{+}^{2}\right)}{r_{c}^{2} r_{+}^{2}}\right)
\end{aligned}
$$




$$
\begin{aligned}
& -\frac{Q^{2}}{4(d-3)\left(r_{c}^{2}+2 \widetilde{\alpha}\right) r_{c}^{2 d-7}} \\
& \cdot\left(2-\frac{(d-1)\left(r_{c}^{2 d-4}-r_{+}^{2 d-4}\right)}{(d-2) r_{+}^{d-3}\left(r_{c}^{d-1}-r_{+}^{d-1}\right)}\right) .
\end{aligned}
$$

The thermodynamic quantities for the two horizons satisfy the first law of black hole thermodynamics [34, 37]

$$
\begin{gathered}
\delta M=\frac{\kappa_{+}}{2 \pi} \delta S_{+}+\Phi_{+} \delta Q+V_{+} \delta P+\widetilde{V}_{+} d \widetilde{\alpha}, \\
\delta M=\frac{\kappa_{c}}{2 \pi} \delta S_{c}+\Phi_{c} \delta Q+V_{c} \delta P+\widetilde{V}_{c} d \widetilde{\alpha},
\end{gathered}
$$

where

$$
\begin{gathered}
S_{+}=\frac{\sum_{k} r_{+}^{(d-2)}}{4}\left(1+\frac{2(d-2) \tilde{\alpha}}{(d-4) r_{+}^{2}}\right), \\
S_{c}=\frac{\sum_{k} r_{c}^{(d-2)}}{4}\left(1+\frac{2(d-2) \tilde{\alpha}}{(d-4) r_{c}^{2}}\right), \\
\Phi_{+}=\frac{\sum_{k} Q}{16 \pi(d-3) r_{+}^{d-3}}, \\
\Phi_{c}=\frac{\sum_{k} Q}{16 \pi(d-3) r_{c}^{d-3}}, \\
V_{+}=\frac{\Sigma_{k} r_{+}^{d-1}}{d-1}, \\
V_{c}=\frac{\sum_{k} r_{c}^{d-1}}{d-1}, \\
\widetilde{V}_{+}=\frac{(d-2)}{16 \pi} r_{+}^{d-5}, \\
\widetilde{V}_{c}=\frac{(d-2)}{16 \pi} r_{c}^{d-5}, \\
P=-\frac{\Lambda}{8 \pi} .
\end{gathered}
$$

\section{Effective Thermodynamic Quantities of Charged GB Black Hole}

We have given the thermodynamic quantities without considering the relationship between the black hole horizon and the cosmological horizon. Because there are four variables $M$, $Q, \Lambda$, and $\widetilde{\alpha}$ in the spacetime, the thermodynamic quantities corresponding to the black hole horizon and the cosmological horizon are functions of these variables. Through the four variables, there are some connections between the thermodynamic quantities corresponding to the black hole horizon and the ones corresponding to the cosmological horizon. When the thermodynamic property of charged de Sitter spacetime is studied, we must consider the relationship with the two horizons. Recently, by studying Hawking radiation of de Sitter spacetime, [50-52] obtained that the outgoing rate of the charged de Sitter spacetime which radiates particles with energy $\omega$ is

$$
\Gamma=e^{\Delta S_{+}+\Delta S_{c}},
$$

where $\Delta S_{+}$and $\Delta S_{c}$ are Bekenstein-Hawking entropy difference corresponding to the black hole horizon and the cosmological horizon after charged de Sitter spacetime radiates particles with energy $\omega$. Therefore, the thermodynamic entropy of the charged de Sitter spacetime is the sum of the black hole horizon entropy and the cosmological horizon entropy:

$$
S=S_{+}+S_{c} \text {. }
$$

Recently, thermodynamic volume of charged dS black hole is given by $[34,37]$

$$
V=V_{c}-V_{+}
$$

From (9), one can obtain

$$
\begin{aligned}
& d S_{+}=\frac{\sum_{k}(d-2)}{4} r_{+}^{d-5}\left(r_{+}^{2}+2 \tilde{\alpha}\right) d r_{+}, \\
& d S_{c}=\frac{\sum_{k}(d-2)}{4} r_{c}^{d-5}\left(r_{c}^{2}+2 \tilde{\alpha}\right) d r_{c} .
\end{aligned}
$$

Substituting (12) and (13) into (14), we obtain

$$
\begin{aligned}
d S_{+}= & \frac{r_{c}^{3}\left(r_{+}^{2}+2 \tilde{\alpha}\right) d S}{r_{c}^{3}\left(r_{+}^{2}+2 \widetilde{\alpha}\right)+r_{+}^{3}\left(r_{c}^{2}+2 \tilde{\alpha}\right)} \\
& -\frac{(d-2)\left(r_{+}^{2}+2 \tilde{\alpha}\right)\left(r_{c}^{2}+2 \tilde{\alpha}\right) d V}{4\left[r_{c}^{3}\left(r_{+}^{2}+2 \tilde{\alpha}\right)+r_{+}^{3}\left(r_{c}^{2}+2 \widetilde{\alpha}\right)\right]}, \\
d S_{c}= & \frac{r_{+}^{3}\left(r_{c}^{2}+2 \tilde{\alpha}\right) d S}{r_{c}^{3}\left(r_{+}^{2}+2 \widetilde{\alpha}\right)+r_{+}^{3}\left(r_{c}^{2}+2 \tilde{\alpha}\right)} \\
& +\frac{(d-2)\left(r_{+}^{2}+2 \tilde{\alpha}\right)\left(r_{c}^{2}+2 \tilde{\alpha}\right) d V}{4\left[r_{c}^{3}\left(r_{+}^{2}+2 \tilde{\alpha}\right)+r_{+}^{3}\left(r_{c}^{2}+2 \widetilde{\alpha}\right)\right]} .
\end{aligned}
$$

Substituting (15) into (8) and (9), we get

$$
d M=T_{\mathrm{eff}} d S-P_{\mathrm{eff}} d V+\Phi_{\mathrm{eff}} d Q+\widetilde{V}_{\mathrm{eff}} d \widetilde{\alpha}
$$

where the effective temperature $T_{\text {eff }}$, effective electric potential $\Phi_{\text {eff }}$, and effective pressure $P_{\text {eff }}$ are, respectively,

$$
\begin{gathered}
T_{\mathrm{eff}}=\frac{\kappa_{+} V_{c} r_{c}^{3}\left(r_{+}^{2}+2 \widetilde{\alpha}\right)-\kappa_{c} V_{+} r_{+}^{3}\left(r_{c}^{2}+2 \tilde{\alpha}\right)}{2 \pi\left(V_{c}-V_{+}\right)\left[r_{c}^{3}\left(r_{+}^{2}+2 \widetilde{\alpha}\right)+r_{+}^{3}\left(r_{c}^{2}+2 \widetilde{\alpha}\right)\right]}, \\
\Phi_{\mathrm{eff}}=\frac{\Phi_{+} V_{c}-\Phi_{c} V_{+}}{V_{c}-V_{+}}, \\
P_{\mathrm{eff}}=\frac{(d-2)\left(r_{+}^{2}+2 \widetilde{\alpha}\right)\left(r_{c}^{2}+2 \widetilde{\alpha}\right)}{8 \pi\left[r_{c}^{3}\left(r_{+}^{2}+2 \widetilde{\alpha}\right)+r_{+}^{3}\left(r_{c}^{2}+2 \widetilde{\alpha}\right)\right]} \frac{\left(\kappa_{+} V_{c}+\kappa_{c} V_{+}\right)}{\left(V_{c}-V_{+}\right)}, \\
\widetilde{V}_{\mathrm{eff}}=\frac{V_{c} \widetilde{V}_{+}-V_{+} \widetilde{V}_{c}}{V_{c}-V_{+}} .
\end{gathered}
$$


Substituting (7) and (10) into (17), we obtain

$$
\begin{aligned}
& T_{\text {eff }} \\
& =\frac{x\left[(d-1)\left(1-x^{2}\right)\left(1+x^{d-1}\right)-2\left(1-x^{d+1}\right)\right]}{4 \pi r_{c}\left(1-x^{d-1}\right)\left[\left(x^{2}+2 \tilde{\alpha} / r_{c}^{2}\right)+x^{3}\left(1+2 \tilde{\alpha} / r_{c}^{2}\right)\right]} \\
& +\frac{\tilde{\alpha}\left[(d-1)\left(1-x^{2}\right)\left(1+x^{d-1}\right)\left(1+x^{2}\right)-4\left(1-x^{d+3}\right)\right]}{4 \pi r_{c}^{3} x\left(1-x^{d-1}\right)\left[\left(x^{2}+2 \tilde{\alpha} / r_{c}^{2}\right)+x^{3}\left(1+2 \tilde{\alpha} / r_{c}^{2}\right)\right]} \\
& +\frac{Q^{2}}{8 \pi(d-3)(d-2)} \\
& \quad \cdot \frac{(d-1)\left(1+x^{d-1}\right)\left(1-x^{2 d-4}\right)-2(d-2)\left(1-x^{3 d-5}\right)}{r_{c}^{2 d-5} x^{2 d-7}\left(1-x^{d-1}\right)\left[\left(x^{2}+2 \tilde{\alpha} / r_{c}^{2}\right)+x^{3}\left(1+2 \widetilde{\alpha} / r_{c}^{2}\right)\right]},
\end{aligned}
$$

$P_{\text {eff }}$

$$
\begin{aligned}
& =-\frac{(d-2)\left(x^{2}+2 \tilde{\alpha} / r_{c}^{2}\right)\left(1+2 \widetilde{\alpha} / r_{c}^{2}\right)\left(1+x^{d}\right)}{4 \pi r_{c}^{2} x\left(1-x^{d-1}\right)\left[\left(x^{2}+2 \tilde{\alpha} / r_{c}^{2}\right)+x^{3}\left(1+2 \tilde{\alpha} / r_{c}^{2}\right)\right]} \\
& +\frac{(d-2)(d-1)\left(1-x^{2}\right) x}{8 \pi r_{c}^{2}\left(1-x^{d-1}\right)^{2}} \\
& \cdot\left(\frac{\left(1+x^{2 d-3}\right)+2 \widetilde{\alpha}\left(1+x^{2 d-5}\right) / r_{c}^{2}}{\left(x^{2}+2 \widetilde{\alpha} / r_{c}^{2}\right)+x^{3}\left(1+2 \widetilde{\alpha} / r_{c}^{2}\right)}\right) \\
& +\frac{\tilde{\alpha}(d-2)(d-1)\left(1-x^{2}\right)\left(1+x^{2}\right)}{8 \pi r_{c}^{4}\left(1-x^{d-1}\right)^{2} x} \\
& \cdot\left(\frac{\left(1+x^{2 d-3}\right)+2 \widetilde{\alpha}\left(1+x^{2 d-5}\right) / r_{c}^{2}}{\left(x^{2}+2 \widetilde{\alpha} / r_{c}^{2}\right)+x^{3}\left(1+2 \widetilde{\alpha} / r_{c}^{2}\right)}\right) \\
& -\frac{Q^{2}(d-2)}{8 \pi(d-3) r_{c}^{2 d-4} x^{2 d-7}\left(1-x^{d-1}\right)} \\
& \cdot\left(\frac{\left(1+x^{3 d-6}\right)+2 \widetilde{\alpha}\left(1+x^{3 d-8}\right) / r_{c}^{2}}{\left(x^{2}+2 \widetilde{\alpha} / r_{c}^{2}\right)+x^{3}\left(1+2 \widetilde{\alpha} / r_{c}^{2}\right)}\right) \\
& +\frac{Q^{2}(d-1)\left(1-x^{2 d-4}\right)}{16 \pi(d-3) r_{c}^{2 d-4} x^{2 d-7}\left(1-x^{d-1}\right)^{2}} \\
& \cdot\left(\frac{\left(1+x^{2 d-3}\right)+2 \widetilde{\alpha}\left(1+x^{2 d-5}\right) / r_{c}^{2}}{\left(x^{2}+2 \widetilde{\alpha} / r_{c}^{2}\right)+x^{3}\left(1+2 \widetilde{\alpha} / r_{c}^{2}\right)}\right) \text {, }
\end{aligned}
$$$$
\Phi_{\mathrm{eff}}=\frac{\Sigma_{k} Q\left(1-x^{2 d-4}\right)}{16 \pi(d-3) r_{c}^{d-3} x^{d-3}\left(1-x^{d-1}\right)},
$$

where $x:=r_{+} / r_{c}$ and $0<x<1$.

In the case of neglecting the connections between the two horizons, the black hole horizon and the cosmological horizon are two independent thermodynamic systems. Due to the different horizon temperatures, the spacetime cannot

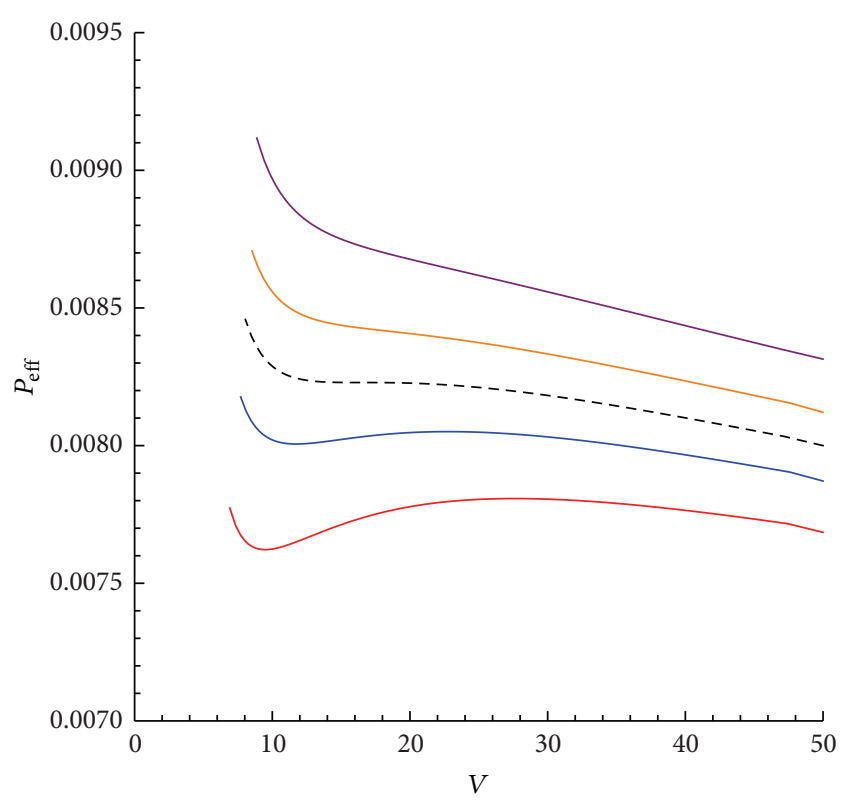

FIgURE 1: $P_{\mathrm{eff}^{-}}-V$ (here $V$ is the thermodynamic volume) diagram of charged GB-dS black hole with $Q=1, d=5, \widetilde{\alpha}=0.1$, and $T_{c}=$ 0.028628 .

be in thermal equilibrium. After considering the connections between both horizons, from (18), one can see that only one effective temperature $T_{\text {eff }}$ left.

\section{Phase Transition in GB-dS Black Hole Spacetime}

Recently, there are many works on the phase transition of black hole thermodynamic system. One can also compare the critical behaviors of van der Waals liquid-gas system and black hole system. Nevertheless, in dS spacetime, there are the cosmological horizon and the black hole horizon. Generally, equilibrium cannot be achieved unless for extremal black hole. In this section, we employe the effective thermodynamic quantities to study the phase transition and critical behaviors of charged GB-dS black hole. This may avoid the apprehension of equilibrium of the black hole system.

Compared with the van der Waals equation,

$$
\left(P+\frac{a}{v^{2}}\right)(v-\widetilde{b})=k T .
$$

Here, $v=V / N$ is the specific volume of the fluid, $P$ is its pressure, $T$ is its temperature, and $k$ is the Boltzmann constant. $a$ is a measure for the attraction between the particles, and $\widetilde{b}$ is the average volume excluded from $v$ by a particle. Similar to [53], for the GB-dS black hole, we can set the specific volume as

$$
v=r_{c}(1-x) .
$$

According to (19), one can employ the two equations

$$
\left(\frac{\partial P_{\mathrm{eff}}}{\partial x}\right)_{T_{\mathrm{eff}}}=0, \quad\left(\frac{\partial^{2} P_{\mathrm{eff}}}{\partial x^{2}}\right)_{T_{\mathrm{eff}}}=0,
$$


TABLE 1: Numerical solutions for $T_{\text {eff }}^{c}, P_{\text {eff }}^{c}$ with fixed $Q=1$ and spacetime dimension $d=5,6,7$ and GB parameter $\widetilde{\alpha}=0.1,0.01,0.001$, respectively.

\begin{tabular}{|c|c|c|c|c|c|c|}
\hline & \multicolumn{3}{|c|}{$T_{\text {eff }}^{c}$} & \multicolumn{3}{|c|}{$P_{\mathrm{eff}}^{c}$} \\
\hline & $d=5$ & $d=6$ & $d=7$ & $d=5$ & $d=6$ & $d=7$ \\
\hline$\widetilde{\alpha}=0.1$ & 0.028628 & 0.040562 & 0.049202 & 0.008229 & 0.018877 & 0.031920 \\
\hline$\widetilde{\alpha}=0.01$ & 0.033651 & 0.045736 & 0.054251 & 0.010356 & 0.021471 & 0.034484 \\
\hline$\widetilde{\alpha}=0.001$ & 0.034297 & 0.046385 & 0.054889 & 0.010632 & 0.021802 & 0.034836 \\
\hline
\end{tabular}
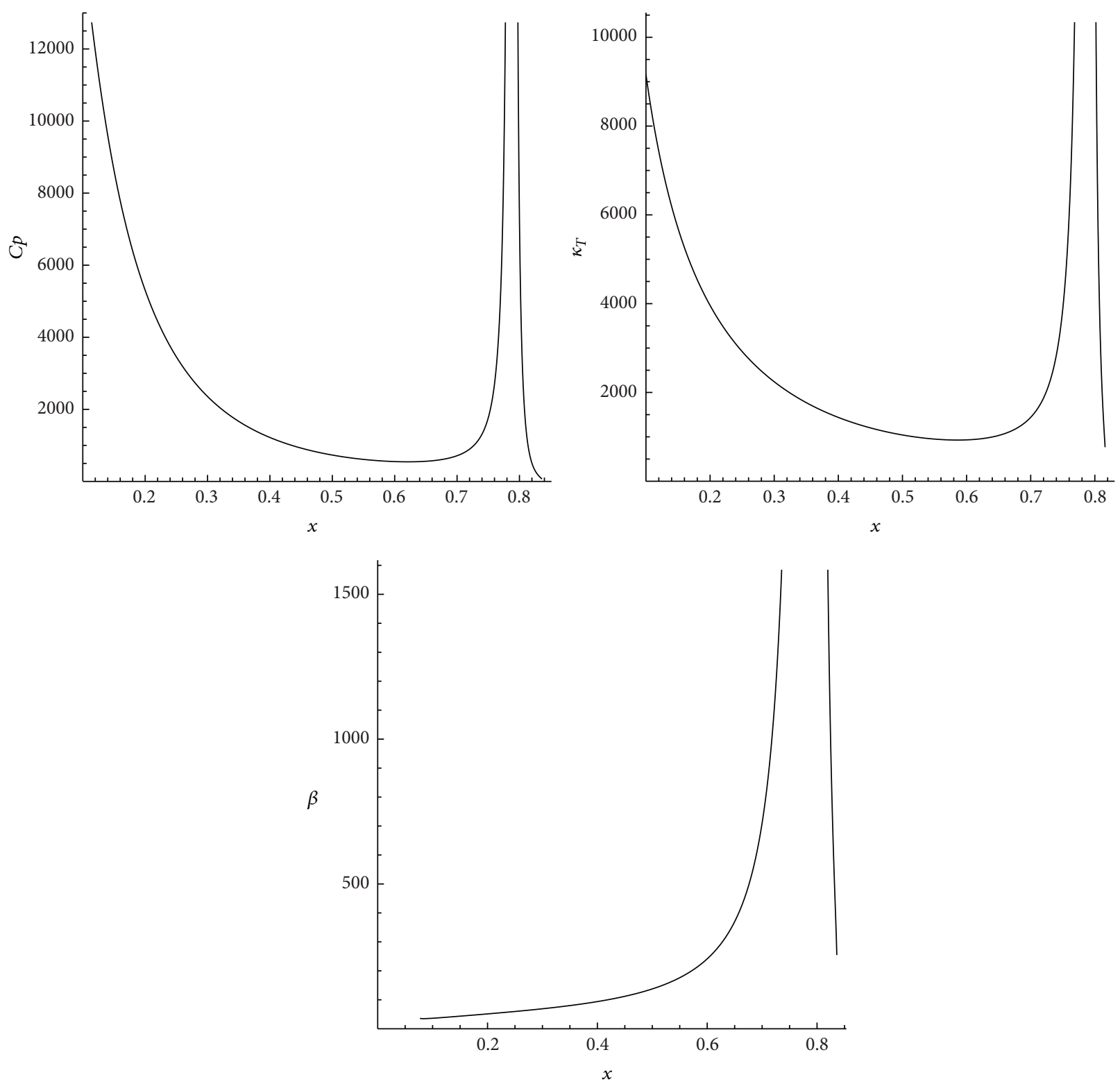

FIgURE 2: $C_{P}-x, \kappa_{T}-x$ and $\beta-x$ curves for charged GB-dS black hole with $Q=1, d=5$, and $\widetilde{\alpha}=0.1$.

to calculate the critical temperature, critical pressure, and the critical specific volume. We can ascertain the critical values $\left(r_{c}^{0}, x^{0}\right)$ for given $Q, d$, and $\widetilde{\alpha}$. According to these values, we can obtain the critical temperature, critical electric potential, critical pressure, and critical volume. As it is shown in Table 1, the critical temperature and critical pressure increase with the dimension of spacetime for fixed $Q$ and $\tilde{\alpha}$. However, they decrease with the increase of the GB parameter $\widetilde{\alpha}$ for fixed spacetime dimension and electric charge $Q$.

In order to describe the relation of $P_{\text {eff }}$ and $V$ in the vicinity of critical temperature, we plot the curves of $P_{\text {eff- }}-V$ at different temperatures, as shown in Figure 1. When 


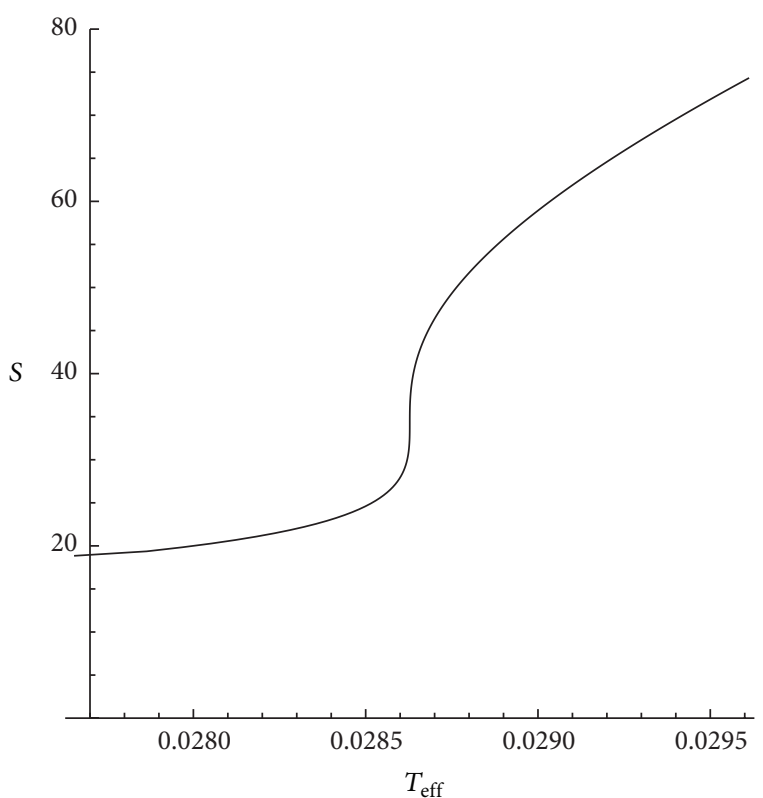

FIgURE 3: $S$ - $T_{\text {eff }}$ curves for charged GB-dS black hole with $Q=1$, $d=5$, and $\widetilde{\alpha}=0.1$.

the effective temperature $T_{\text {eff }}>T_{\text {eff }}^{c}$, the stable condition $(\partial P / \partial V)_{T_{\text {eff }}}<0$ can be satisfied. In the case of $T_{\text {eff }}<T_{\text {eff }}^{c}$, there exists a portion in the curve, where $(\partial P / \partial V)_{T_{\text {eff }}}>0$; thus, in these parts, the system is unstable. So, phase transition may occur only at $T_{\text {eff }}=T_{\text {eff }}^{c}$.

According to Ehrenfest classification, when the chemical potential and its first derivative are continuous, whereas the second derivative of chemical potential is discontinuous, this kind of phase transition is called the second-order phase transition. For van der Waals equation, at the critical point, no latent and sudden change in volume happen between liquid phase and gas phase. According to Ehrenfest classification, this kind of phase transition is continuous.

To understand the types of the phase transition for the GB-dS black hole, we should calculate the specific heat at constant pressure $C_{P}$, the volume expansivity $\beta$, and the isothermal compressibility $\kappa$ :

$$
\begin{gathered}
C_{P}=T_{\mathrm{eff}}\left(\frac{\partial S}{\partial T_{\mathrm{eff}}}\right)_{P_{\mathrm{eff}}}=T_{\mathrm{eff}}\left(\frac{\partial S}{\partial x} \frac{\partial x}{\partial T_{\mathrm{eff}}}\right)_{P_{\mathrm{eff}}}, \\
\beta=\frac{1}{v}\left(\frac{\partial v}{\partial T_{\mathrm{eff}}}\right)_{P_{\mathrm{eff}}}=\frac{1}{v}\left(\frac{\partial v}{\partial x} \frac{\partial x}{\partial T_{\mathrm{eff}}}\right)_{P_{\mathrm{eff}}}, \\
\kappa_{T}=-\frac{1}{v}\left(\frac{\partial v}{\partial P_{\mathrm{eff}}}\right)_{T_{\mathrm{eff}}}=-\frac{1}{v}\left(\frac{\partial v}{\partial x} \frac{\partial x}{\partial P_{\mathrm{eff}}}\right)_{T_{\mathrm{eff}}},
\end{gathered}
$$

where $v$ is the specific volume.

We also depict the curves of $C_{P^{-}} x, \kappa_{T}-x$ and $\beta-x$ in Figure 2, respectively. From these curves, we find that, for the charged GB-dS black hole, there exists an infinite peak in these curves. Moreover, in Figure 3, one can see that the curves of $S-T_{\text {eff }}$ are continuous at the critical point. According to Ehrenfest, the phase transition of the charged GB-dS black hole should be the second-order one, which is similar to the RN-(A)dS black hole.

\section{Discussion and Conclusion}

In this paper, we adopted Ehrenfests classification to study the phase transition of the charged GB-dS black hole. After introducing the connection between the thermodynamic quantities corresponding to the black hole horizon and the cosmological horizon, we give the effective thermodynamic quantities, such as effective pressure $P_{\text {eff }}$, effective temperature $T_{\text {eff }}$ of the black hole. From the equation of the state described by the effective thermodynamic quantities, we study the $P-V$ criticality. As illustrated in Figure 1, it exhibits a similar phase transition to van der Waals equation.

By treating the effective cosmological constant as the thermodynamic pressure, in the extended phase space, we completely follow the Ehrenfest classification to explore the type of the phase transition of the charged GB-dS black hole. It is shown that the entropy is a continuous function of temperature, however, the heat capacity $C_{P}$, the isothermal compressibility $\kappa_{T}$, and the expansion coefficient $\beta$ are all divergent at the critical point. This means this kind of phase transition for the charged GB-dS black hole is second order.

\section{Conflict of Interests}

The authors declare that there is no conflict of interests regarding the publication of this paper.

\section{Acknowledgments}

This work is supported by NSFC under Grant nos. (11475108, 11175109; 11075098; and 11205097) and the doctoral Sustentation foundation of Shanxi Datong University (2011-B-03).

\section{References}

[1] J. D. Bekenstein, "Extraction of energy and charge from a black hole," Physical Review D, vol. 7, no. 4, pp. 949-953, 1973.

[2] J. M. Bardeen, B. Carter, and S. W. Hawking, "The four laws of black hole mechanics," Communications in Mathematical Physics, vol. 31, pp. 161-170, 1973.

[3] S. W. Hawking, "Black hole explosions?" Nature, vol. 248, no. 5443, pp. 30-31, 1974.

[4] S. W. Hawking, "Particle creation by black holes," Communications in Mathematical Physics, vol. 43, no. 3, pp. 199-220, 1975.

[5] S. W. Hawking and D. N. Page, "Thermodynamics of black holes in anti-de Sitter space," Communications in Mathematical Physics, vol. 87, no. 4, pp. 577-588, 1983.

[6] A. Chamblin, R. Emparan, C. V. Johnson, and R. C. Myers, "Charged AdS black holes and catastrophic holography," Physical Review D, vol. 60, no. 6, 1999.

[7] A. Chamblin, R. Emparan, C. V. Johnson, and R. C. Myers, "Holography, thermodynamics, and fluctuations of charged AdS black holes," Physical Review D, vol. 60, no. 10, 20 pages, 1999. 
[8] A. Sahay, T. Sarkar, and G. Sengupta, "Thermodynamic geometry and phase transitions in Kerr-Newman-AdS black holes," Journal of High Energy Physics, vol. 2010, article 118, 2010.

[9] A. Sahay, T. Sarkar, and G. Sengupta, "On the thermodynamic geometry and critical phenomena of AdS black holes," Journal of High Energy Physics, no. 7, article 082, 44 pages, 2010.

[10] A. Sahay, T. Sarkar, and G. Sengupta, "On the phase structure and thermodynamic geometry of R-charged black holes," Journal of High Energy Physics, vol. 2010, article 125, 2010.

[11] R. Banerjee, S. Ghosh, and D. Roychowdhury, "New type of phase transition in Reissner Nordström-AdS black hole and its thermodynamic geometry," Physics Letters, Section B: Nuclear, Elementary Particle and High-Energy Physics, vol. 696, no. 1-2, pp. 156-162, 2011.

[12] R. Banerjee, S. K. Modak, and S. Samanta, "Second order phase transition and thermodynamic geometry in Kerr-AdS black holes," Physical Review D, vol. 84, no. 6, Article ID 064024, 2011.

[13] R. Banerjee and D. Roychowdhury, "Thermodynamics of phase transition in higher dimensional AdS black holes," Journal of High Energy Physics, vol. 2011, article 4, 2011.

[14] R. Banerjee and D. Roychowdhury, "Critical phenomena in Born-Infeld AdS black holes," Physical Review D, vol. 85, no. 4, Article ID 044040, 2012.

[15] R. Banerjee and D. Roychowdhury, "Critical behavior of BornInfeld AdS black holes in higher dimensions," Physical Review D: Particles, Fields, Gravitation and Cosmology, vol. 85, no. 10, Article ID 104043, 2012.

[16] A. Lala and D. Roychowdhury, "Ehrenfest's scheme and thermodynamic geometry in Born-Infeld AdS black holes," Physical Review D-Particles, Fields, Gravitation and Cosmology, vol. 86, no. 8, Article ID 084027, 2012.

[17] B. R. Majhi and D. Roychowdhury, "Phase transition and scaling behavior of topological charged black holes in Horava-Lifshitz gravity," Classical and Quantum Gravity, vol. 29, no. 24, Article ID 245012, 12 pages, 2012.

[18] D. Kastor, S. Ray, and J. Traschen, "Enthalpy and the mechanics of AdS black holes," Classical and Quantum Gravity, vol. 26, Article ID 195011, 2009.

[19] D. Kastor, S. Ray, and J. Traschen, "Smarr formula and an extended first law for Lovelock gravity," Classical and Quantum Gravity, vol. 27, no. 23, Article ID 235014, 17 pages, 2010.

[20] B. P. Dolan, "The cosmological constant and black-hole thermodynamic potentials," Classical and Quantum Gravity, vol. 28, Article ID 125020, 2011.

[21] S. Gunasekaran, D. Kubiznak, and R. B. Mann, "Extended phase space thermodynamics for charged and rotating black holes and Born-Infeld vacuum polarization," Journal of High Energy Physics, vol. 2012, no. 11, article 110, 2012.

[22] B. P. Dolan, "Compressibility of rotating black holes," Physical Review D, vol. 84, Article ID 127503, 2011.

[23] M. Cvetic, G. W. Gibbons, D. Kubiznak, and C. N. Pope, "Black hole enthalpy and an entropy inequality for the thermodynamic volume," Physical Review D, vol. 84, no. 2, Article ID 024037, 17 pages, 2011.

[24] D. Kubiznak and R. B. Mann, " $P-V$ criticality of charged AdS black holes," Journal of High Energy Physics, vol. 1207, article 33, 2012.

[25] B. P. Dolan, "Where is the $P d V$ in the first law of black hole thermodynamics?" in Open Questions in Cosmology, chapter 12, 2012.
[26] S.-W. Wei and Y.-X. Liu, "Critical phenomena and thermodynamic geometry of charged Gauss-Bonnet AdS black holes," Physical Review D: Particles, Fields, Gravitation and Cosmology, vol. 87, no. 4, Article ID 044014, 2013.

[27] R.-G. Cai, L.-M. Cao, L. Li, and R.-Q. Yang, "P-V criticality in the extended phase space of Gauss-Bonnet black holes in AdS space," Journal of High Energy Physics, vol. 2013, no. 9, article 5, 2013.

[28] W. Xu, H. Xu, and L. Zhao, "Gauss-bonnet coupling constant as a free thermodynamical variable and the associated criticality," The European Physical Journal C, vol. 74, no. 7, article 2970, 2014.

[29] D. C. Zou, Y. Liu, and B. Wang, "Critical behavior of charged Gauss-Bonnet-AdS black holes in the grand canonical ensemble," Physical Review D, vol. 90, no. 4, Article ID 044063, 2014.

[30] S. H. Hendi and M. H. Vahidinia, "Extended phase space thermodynamics and $P-V$ criticality of black holes with a nonlinear source," Physical Review D, vol. 88, no. 8, Article ID 084045, 2013.

[31] R. Zhao, H.-H. Zhao, M.-S. Ma, and L.-C. Zhang, "On the critical phenomena and thermodynamics of charged topological dilaton AdS black holes," The European Physical Journal C, vol. 73, $\mathrm{m}$ article 2645, 2013.

[32] N. Altamirano, D. Kubiznak, and R. Mann, "Reentrant phase transitions in rotating AdS black holes," Physical Review D, vol. 88, no. 10, Article ID 101502, 5 pages, 2013.

[33] S.-W. Wei and Y.-X. Liu, "Triple points and phase diagrams in the extended phase space of charged Gauss-Bonnet black holes in AdS space," Physical Review D, vol. 90, no. 4, Article ID 044057, 2014.

[34] B. P. Dolan, "Vacuum energy and the latent heat of AdS-Kerr black holes," Physical Review D, vol. 90, no. 8, Article ID 084002, 2014.

[35] B. Mirza and Z. Sherkatghanad, "Phase transitions of hairy black holes in massive gravity and thermodynamic behavior of charged AdS black holes in an extended phase space," Physical Review D, vol. 90, no. 8, Article ID 084006, 2014.

[36] R.-G. Cai, "Cardy-Verlinde formula and thermodynamics of black holes in de Sitter spaces," Nuclear Physics B, vol. 628, no. 1-2, pp. 375-386, 2002.

[37] Y. Sekiwa, “Thermodynamics of de Sitter black holes: thermal cosmological constant," Physical Review D, vol. 73, no. 8, Article ID 084009, 2006.

[38] M. Urano, A. Tomimatsu, and H. Saida, "The mechanical first law of black hole spacetimes with a cosmological constant and its application to the Schwarzschild-de SITter spacetime," Classical and Quantum Gravity, vol. 26, no. 10, Article ID 105010, 14 pages, 2009.

[39] D. Kastor and J. Traschen, "Particle production and positive energy theorems for charged black holes in de Sitter," Classical and Quantum Gravity, vol. 13, no. 10, p. 2753, 1996.

[40] L. C. Zhang, H. F. Li, and R. Zhao, "Thermodynamics of the Reissner-Nordström-de Sitter black hole," Science China: Physics, Mechanics and Astronomy, vol. 54, no. 8, pp. 1384-1387, 2011.

[41] B. P. Dolan, D. Kastor, D. Kubizňák, R. B. Mann, and J. Traschen, "Thermodynamic volumes and isoperimetric inequalities for de Sitter black holes," Physical Review D: Particles, Fields, Gravitation and Cosmology, vol. 87, no. 10, 14 pages, 2013.

[42] Y. S. Myung, "Thermodynamics of the Schwarzschild-de Sitter black hole: thermal stability of the Nariai black hole," Physical Review D: Particles, Fields, Gravitation, and Cosmology, vol. 77, no. 10, Article ID 104007, 2008. 
[43] R. G. Cai, "Dark energy and de Sitter spacetime," Physics, vol. 34, p. 555, 2005 (Chinese).

[44] S. Bhattacharya and A. Lahiri, "Mass function and particle creation in Schwarzschild-de Sitter spacetime," The European Physical Journal C, vol. 73, no. 12, pp. 1-10, 2013.

[45] H.-H. Zhao, L.-C. Zhang, M.-S. Ma, and R. Zhao, " $P-V$ criticality of higher dimensional charged topological dilaton de Sitter black holes," Physical Review D, vol. 90, no. 6, Article ID 064018, 2014.

[46] R. G. Cai, J. Y. Ji, and K. S. Soh, "Action and entropy of black holes in spacetimes with a cosmological constant," Classical and Quantum Gravity, vol. 15, no. 9, pp. 2783-2793, 1998.

[47] G. W. Gibbons, H. Lü, D. N. Page, and C. N. Pope, "Rotating black holes in higher dimensions with a cosmological constant," Physical Review Letters, vol. 93, no. 17, Article ID 171102, 2004.

[48] G. W. Gibbons, H. Lu, D. N. Page, and C. N. Pope, "The general Kerr-de Sitter metrics in all dimensions," Journal of Geometry and Physics, vol. 53, no. 1, pp. 49-73, 2005.

[49] R.-G. Cai, "Gauss-Bonnet black holes in AdS spaces," Physical Review D, vol. 65, no. 8, 9 pages, 2002.

[50] R. Zhao, L.-C. Zhang, and H.-F. Li, "Hawking radiation of a Reissner-Nordström-de Sitter black hole," General Relativity and Gravitation, vol. 42, no. 4, pp. 975-983, 2010.

[51] R. Zhao, L. Zhang, H. Li, and Y. Wu, "Hawking radiation of a high-dimensional rotating black hole," The European Physical Journal C: Particles and Fields, vol. 65, no. 1-2, pp. 289-293, 2010.

[52] R. Zhao and L. C. Zhang, "A new explanation for statistical entropy of charged black hole," Science China Physics, Mechanics and Astronomy, vol. 56, no. 9, pp. 1632-1635, 2013.

[53] R. Zhao, M. S. Ma, H. H. Zhao, and L. C. Zhang, "The critical phenomena and thermodynamics of the Reissner-Nordstromde Sitter black hole," Advances in High Energy Physics, vol. 2014, Article ID 124854, 2014. 

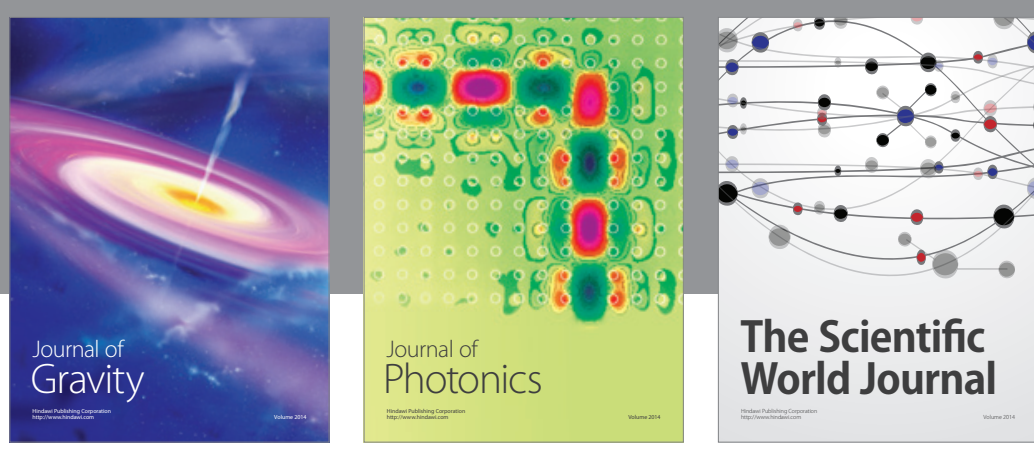

The Scientific World Journal
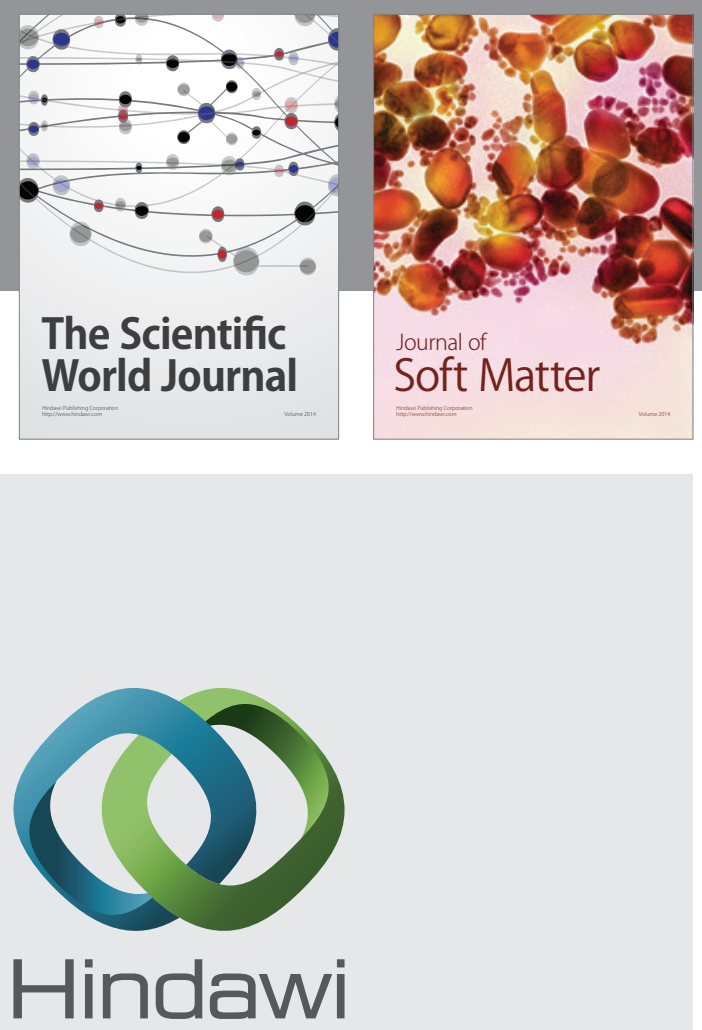

Submit your manuscripts at

http://www.hindawi.com

nternational Journal of

Statistical Mechanics
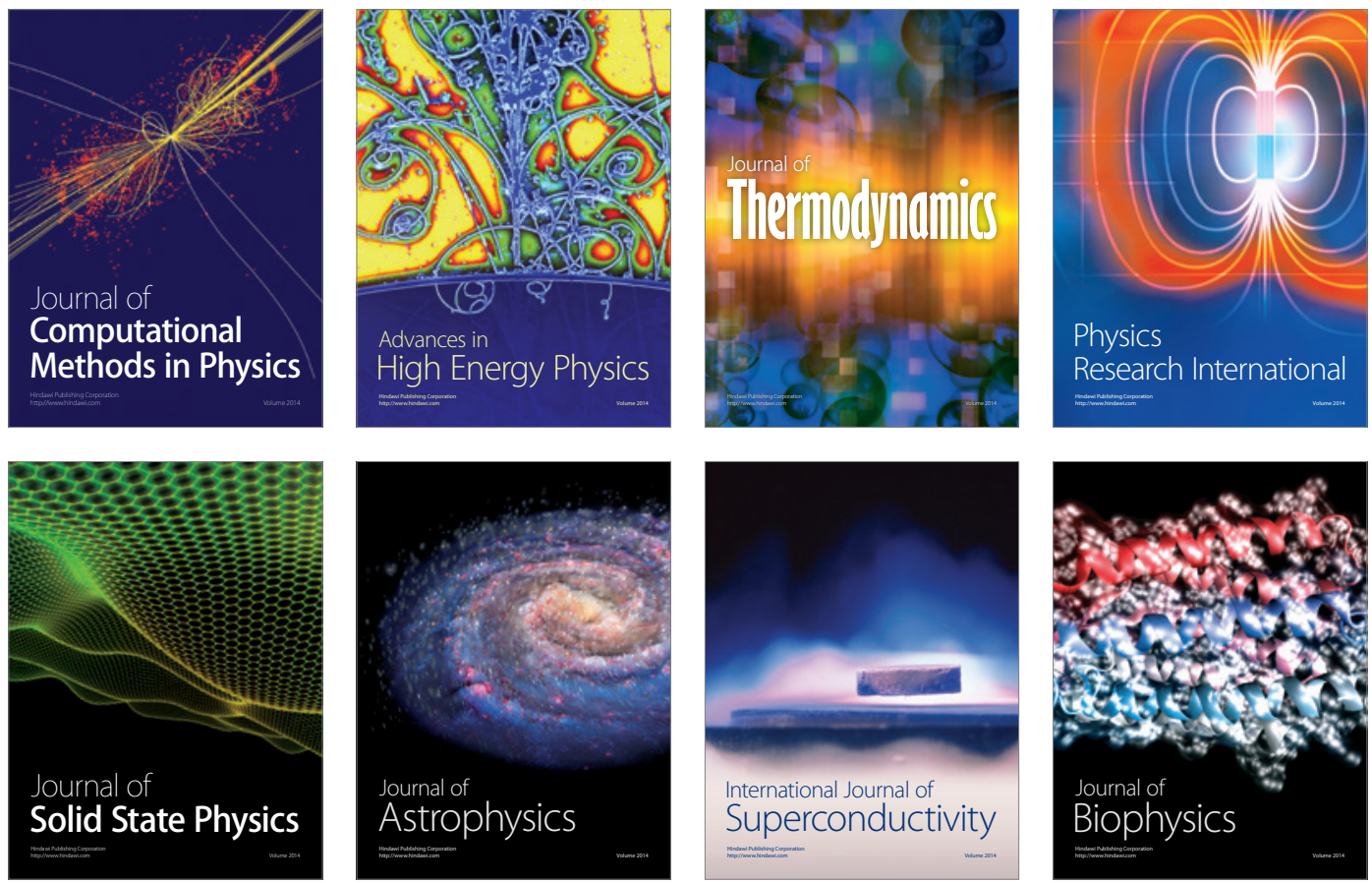
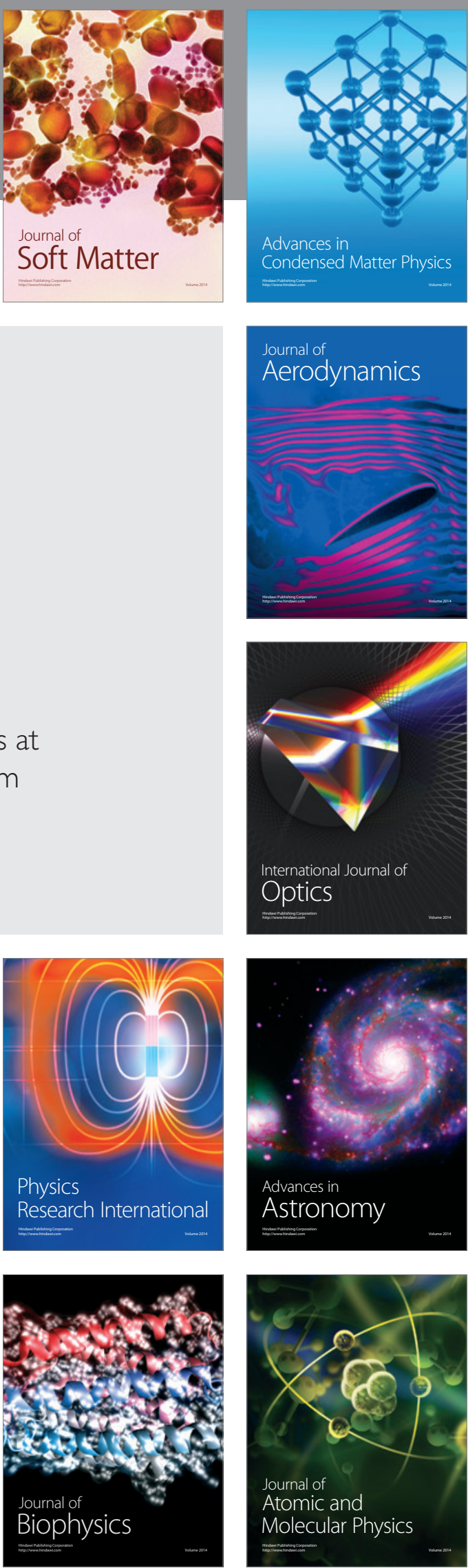